\title{
Sub-threshold investigation of two coupled photonic crystal cavities
}

Schubert, Martin; Frandsen, Lars Hagedorn; Skovgård, Troels Suhr; Lund-Hansen, Toke; Nielsen, Henri Thyrrestrup; Bichler, Max; Finley, Jonathan J.; Lodahl, Peter; Hvam, Jørn Märcher; Yvind, Kresten

Published in:

Technical digest, CLEO/IQEC

Publication date:

2009

Document Version

Publisher's PDF, also known as Version of record

Link back to DTU Orbit

Citation (APA):

Schubert, M., Frandsen, L. H., Skovgård, T. S., Lund-Hansen, T., Nielsen, H. T., Bichler, M., Finley, J. J., Lodahl, P., Hvam, J. M., \& Yvind, K. (2009). Sub-threshold investigation of two coupled photonic crystal cavities. In Technical digest, CLEO/IQEC (pp. 1-2). IEEE.

\section{General rights}

Copyright and moral rights for the publications made accessible in the public portal are retained by the authors and/or other copyright owners and it is a condition of accessing publications that users recognise and abide by the legal requirements associated with these rights.

- Users may download and print one copy of any publication from the public portal for the purpose of private study or research.

- You may not further distribute the material or use it for any profit-making activity or commercial gain

- You may freely distribute the URL identifying the publication in the public portal 


\title{
Sub-threshold investigation
}

\section{of two coupled photonic crystal cavities}

\author{
Martin Schubert $^{1}$, Lars H. Frandsen ${ }^{1}$, Troels Suhr ${ }^{1}$, Toke Lund-Hansen ${ }^{1}$, Henri Thyrrestrup ${ }^{1}$, \\ Max Bichler ${ }^{2}$, Jonathan J. Finley ${ }^{2}$, Peter Lodahl ${ }^{1}$, Jørn M. Hvam ${ }^{1}$, and Kresten Yvind ${ }^{1}$ \\ ${ }^{I}$ DTU Fotonik, Technical University of Denmark, Building 343, DK-2800 Kgs. Lyngby, Denmark \\ marsc@fotonik.dtu.dk \\ ${ }^{2}$ Walter Schottky Institut and Physik Department, Technische Universität München, Am Coulombwall 3, D-85748 Garching, Germany
}

\begin{abstract}
The behavior of two coupled photonic crystal membrane cavities with quantum dots separated by different number of holes is investigated. The measured spectral splitting with increased coupling is verified by $3 \mathrm{D}$ calculations and discussed.

(C)2009 Optical Society of America

OCIS codes: (140.3410) Laser resonators; (230.4555) Coupled resonators; (230.5298) Photonic crystals
\end{abstract}

\section{Introduction}

Coupled photonic crystal (PhC) cavity systems have been of recent interest for lasing applications due to their low threshold powers, high differential quantum efficiencies, and high output powers [1]. However, so far little work has been done on investigating the mode structure of such systems below threshold. The behavior below threshold is important for tailoring and selecting the lasing mode, since modes close to the lasing mode may be detrimental to the performance of the laser and should be suppressed above threshold.

Simulating a large array of coupled $\mathrm{PhC}$ cavities is cumbersome due to the large size of the structure. Also, the mode structures become more complex with increasing number of coupled cavities since such a system exhibits a behavior similar to what the tight-binding model describes for atoms [2,3]. A good starting point for understanding large coupled $\mathrm{PhC}$ arrays is therefore to investigate the coupling of two coupled $\mathrm{PhC}$ cavities separated by different numbers of holes where it is feasible to compare measurements with simulations.

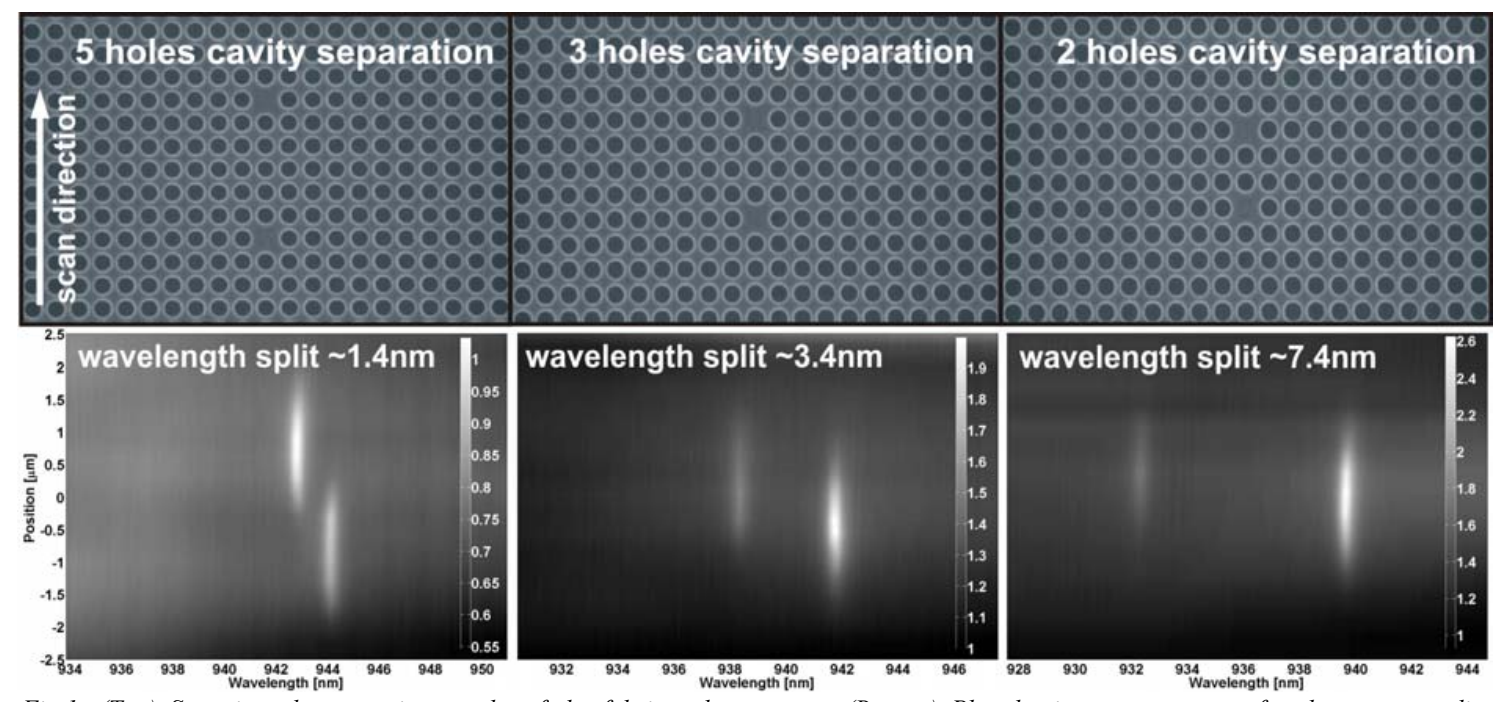

Fig.1: (Top) Scanning electron micrographs of the fabricated structures. (Bottom) Photoluminescence spectra for the corresponding structures at different spatial positions (y-axis) showing the different wavelengths (x-axis).

\section{Measurements}

We have fabricated $\mathrm{PhC}$ structures in a $\sim 160 \mathrm{~nm}$ thick GaAs membrane by arranging air holes with diameter $\mathrm{d} \approx 210 \mathrm{~nm}$ in a quadratic lattice with pitch a=280 nm. PhC structures with two coupled cavities defined in the $\mathrm{PhC}$ by single missing holes and separated by two, three and five holes have been characterized. Scanning electron micrograph (SEM) pictures of the fabricated structures can be seen in Fig.1 (top). Confirmed by planewave-expansion and finite-difference time-domain calculations quadrupole and dipole modes were measured using photoluminescence (PL) measurements of InGaAs quantum dots embedded in the PhC membrane. The fabricated coupled cavities were scanned along the direction of the coupling axis with a collection spot-size of $\sim 1.5 \mu \mathrm{m}$ and several PL-spectra were recorded for each coupled system. The spectra for the quadrupole mode can be seen in Fig.1 (bottom). A clear spectral splitting of $\sim 7.4 \mathrm{~nm}$ and $\sim 3.4 \mathrm{~nm}$ for the quadrupole mode in two coupled cavities separated by 2 and 3 holes, respectively, are found. The spectral 


\section{CFE7.pdf}

splitting is a consequence of the different field distributions in the coupling region between the cavities for the even and odd parity modes of the coupled system. The parity of the low and high wavelength mode can be engineered by selecting either an odd or even number of holes between the cavities. This can be understood by inspecting how the fields distribute in air and semiconductor material between cavities. Similar results for a different cavity system have been reported in Ref. [3].

Different polarizations were measured for each structure to further investigate the mode structure of the coupled system. By scanning the membrane and analyzing the spectra, the different modes could be resolved spatially. The spatial separation of the PL peaks for the two cavities separated by five holes corresponds nicely to the geometrical distance between the two cavities. However, for the two cavities separated by two and three holes, the spatial separation is smaller than the actual distance. This combined with the large spectral splitting strongly indicates that the peaks for the two and three hole cavity distances originate from a coupled mode while the two cavities separated by five holes seem to be mostly decoupled. This is further supported by looking at the polarization behavior of the peaks (not shown).

\section{Simulations and Conclusion}

The experimental results are verified by using 3D finite-difference time-domain (FDTD) calculations showing good agreement between the calculated spectral splitting of $\sim 8.2 \mathrm{~nm}(\sim 4.4 \mathrm{~nm})$ and the measured splitting of $\sim 7.4 \mathrm{~nm}(\sim 3.4 \mathrm{~nm})$ for 2 (3) holes separation, respectively, as shown in Fig. 2 . The deviations are due to the limited spatial resolution in the FDTD calculation and uncertainties in the parameters of the fabricated $\mathrm{PhC}$. We believe that the cavity system separated by five holes is practically decoupled and causes the above mentioned measured spatial separation. The small $(\sim 1.4 \mathrm{~nm})$ spectral splitting is due to low fabrication tolerances and the lack of proximity correction.

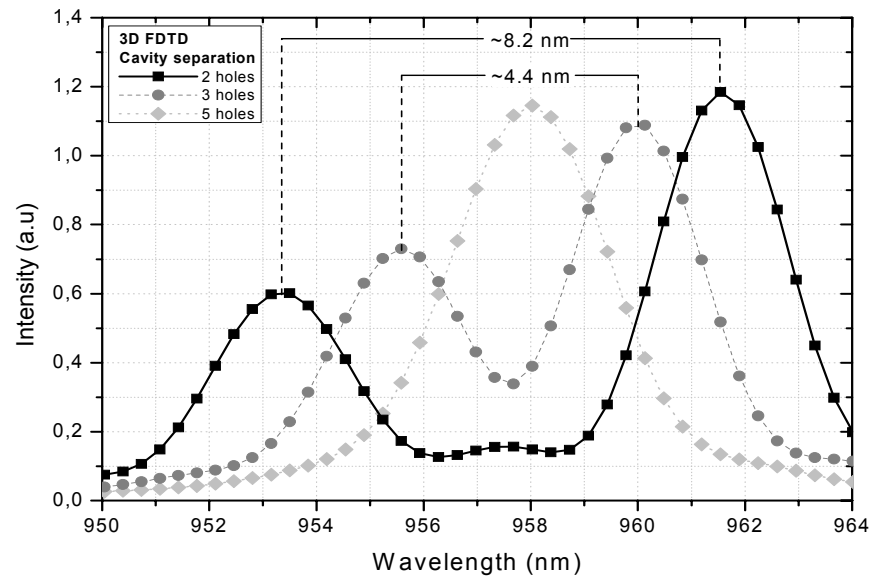

Fig. 2: Finite-difference time-domain simulations of two coupled cavities for different cavity separations.

We think these results are crucial for the further investigation and understanding of coupled cavity lasers and for achieving a true single-wavelength coupled $\mathrm{PhC}$ resonator array laser. We have measured how the coupling affects the mode splitting and thereby gained information on how the coupling strength depends on the number of holes in between the cavities. Further investigations into the far-field emission of these structures as well as possibilities of tuning the coupling between cavities beyond adding or removing complete holes are underway.

\section{References}

[1] H. Altug et. al., Opt. Express 13, pp. 8819-8828, (2005).

[2] M. Notomi et. al., Nature Photonics 2, pp. 741-747, (2008).

[3] K. A. Atlasov et. al., Opt. Express 16, pp. 16255-16264, (2008).

\section{Acknowledgments}

This work was supported in part by the Danish Technical Research Council through the research program 'Coupled photonic crystal resonator array laser' (CORAL) and in part by the 'Nanosystems Initiative Munich' (NIM) 